\title{
SOME INCLUSION RELATIONS BETWEEN MATRICES COMPOUNDED FROM CESARO MATRICES
}

\author{
BY \\ A. J. WHITE
}

1. Introduction. Let $\mathscr{P}$ denote the family of lower triangular matrices which define regular sequence to sequence transformations and which have nonnegative elements and nonzero elements on the leading diagonal; i.e. $B=\left(b_{n k}\right) \in \mathscr{P}$ if and only if $b_{n k} \geqq 0(n=0,1, \cdots ; k=0,1, \cdots), b_{n k}=0(n<k), b_{n n}>0(n=0,1, \cdots)$ and $b_{n k} \rightarrow 0 \quad(n \rightarrow \infty, k=0,1, \cdots)$,

$$
\lim _{n \rightarrow \infty} \sum_{k=0}^{n} b_{n k}=1
$$

Let $\{A(r)\}(r=1,2, \cdots)$ be any sequence of infinite matrices. If $A(r)=\left(a_{n k}(r)\right)$ $(r=1,2, \cdots)$ and if $\left\{r_{n}\right\}$ is any sequence of positive integers, the matrix $A\left\{r_{n}\right\}=\left(a_{n k}\left(r_{n}\right)\right)$, which has as its $n$th row the $n$th row of $A\left(r_{n}\right)$, is said to be compounded from the sequence $\left\{A\left(r_{n}\right)\right\}$ or to be a compounded matrix.

The results set out in the following theorem concern some properties of compounded matrices.

THEOREM A. (i) If $A(r) \in \mathscr{P}(r=1,2, \cdots)$ then there is an increasing sequence $\left\{R_{n}\right\}$ of positive integers such that if $1 \leqq r_{n} \leqq R_{n}$ the compounded matrix $A\left\{r_{n}\right\}$ is regular.

(ii) If $A(r) \in \mathscr{P}$ and $A(r+1)(A(r))^{-1} \in \mathscr{P}(r=1,2, \cdots)$, if $\left\{r_{n}\right\}$ and $\left\{r_{n}^{\prime}\right\}$ are sequences of positive integers such that $A\left\{r_{n}\right\}$ and $A\left\{r_{n}^{\prime}\right\}$ are regular and if $r_{n}^{\prime} \leqq r_{n}$ for all sufficiently large values of $n$ then $\left({ }^{1}\right) A\left\{r_{n}^{\prime}\right\} \subseteq A\left\{r_{n}\right\}$.

(iii) If $A(r) \in \mathscr{P}$ and $A(r)(A(r+1))^{-1} \in \mathscr{P}(r=1,2, \cdots)$, if $\left\{r_{n}\right\}$ and $\left\{r_{n}^{\prime}\right\}$ are sequences of positive integers such that $A\left\{r_{n}\right\}$ and $A\left\{r_{n}^{\prime}\right\}$ are regular and if $r_{n}^{\prime} \leqq r_{n}$ for all sufficiently large values of $n$ then $A\left\{r_{n}^{\prime}\right\} \subseteq A\left\{r_{n}\right\}$.

Of these results $(\mathrm{i})\left({ }^{2}\right)$ and (ii) are due to Agnew ([1], Theorems 3.1 and 3.2 and the remarks in $\$ 5$; cf. also the references given there) and (iii) may be obtained by making simple changes in the arguments used to prove (ii).

Now suppose that $A(r) \in \mathscr{P}(r=1,2, \cdots)$ that $A(1) \subseteq A(2) \subseteq \cdots$ (this is certainly

Received by the editors August 5, 1963.

(1) Throughout this note we write $A \subseteq B$ if $s_{n} \rightarrow s(A)$ implies $s_{n} \rightarrow s(B)$, and $A \subset B$ if $A \subseteq B$ but there is a sequence $\left\{s_{n}\right\}$ such that $s_{n} \rightarrow s(B)$ but $s_{n} \rightarrow s(A)$. If $A \subseteq B$ and $B \supseteq A$ we write $A \equiv B$.

(2) This result is stated in [1] under the additional hypothesis $A(r+1)(A(r))^{-1} \in \mathscr{P}$ but inspection of the proof shows that this condition is not in fact used. 
the case if $\left.A(r+1)(A(r))^{-1} \in \mathscr{P}\right)$ and that $A(r) \subseteq B(r=1,2, \cdots)$ where $B$ is some regular matrix. It is natural to inquire what relation exists, if any, between $B$ and a compounded matrix $A\left\{r_{n}\right\}$. If $A\left\{r_{n}\right\}$ is regular an attractive conjecture is that $A\left\{r_{n}\right\} \subseteq B$. This can fail to happen in a rather spectacular way, as the following example shows. For $r=1,2, \cdots$ let $A(r)$ be the matrix which transforms a sequence $\left\{s_{n}\right\}$ into the sequence $\left\{t_{n}\right\}$ where

$$
t_{n}=r^{-1} s_{n}+\left(1-r^{-1}\right)(n+1)^{-1} \sum_{v=0}^{n} s_{v} .
$$

Then each $A(r)$ is a Mercerian matrix [5, p. 104] and is equivalent to convergence so that trivially $A(1) \subseteq A(2) \subseteq \cdots \subset C(\alpha), 0<\alpha \leqq 1$. If $r_{n}=n$ the compounded matrix $A\left\{r_{n}\right\}$ transforms $\left\{s_{n}\right\}$ into $\left\{t_{n}^{\prime}\right\}$ where

$$
t_{n}^{\prime}=n^{-1} s_{n}+\left(1-n^{-1}\right)(n+1)^{-1} \sum_{v=0}^{n} s_{v}
$$

and so $A\left\{r_{n}\right\}$ is regular. On the other hand if $s_{n} \rightarrow s C(1)$ then $(n+1)^{-1} \sum_{v=0}^{n} s_{v} \rightarrow s$ and $\left[5\right.$, p. 101] $s_{n}-s=o(n)$. It follows that $n^{-1} s_{n}=o(1)$ and hence that $t_{n}^{\prime} \rightarrow s$ as $n \rightarrow \infty$. Consequently $C(\alpha) \subset A\left\{r_{n}\right\}$ for $0<\alpha<1$.

In this example the condition $A(r+1)(A(r))^{-1} \in \mathscr{P}$ is not fulfilled, but, as we shall see below (Theorem 4), even if it is there may still be a regular compounded matrix $A\left\{r_{n}\right\}$ such that $B \subset A\left\{r_{n}\right\}$.

Throughout the rest of this note we consider matrices compounded from Cesaro matrices. We write $\left({ }^{3}\right)$

$$
\varepsilon_{n}(\alpha)=\frac{(\alpha+1)(\alpha+2) \cdots(\alpha+n)}{n !}
$$

so that, for $\alpha>-1, C(\alpha)$, the Cesaro matrix of order $\alpha$, is the lower triangular matrix $\left(a_{n k}\right)$ where $a_{n k}=\varepsilon_{n-k}(\alpha-1) / \varepsilon_{n}(\alpha)$. If $\alpha_{n}>-1(n=0,1, \cdots)$ then $C\left\{\alpha_{n}\right\}$ denotes the lower triangular compounded matrix $\left(b_{n k}\right)$ where $b_{n k}=\varepsilon_{n-k}\left(\alpha_{n}-1\right) / \varepsilon_{n}\left(\alpha_{n}\right)$.

It is well known and easily verified that if $\alpha_{n} \geqq 0(n=0,1, \cdots)$ then $C\left\{\alpha_{n}\right\}$ is regular if and only if $\alpha_{n}=o(n)$ as $n \rightarrow \infty$.

Agnew [1, Theorem 6.1] has studied the relation between $C\left\{\alpha_{n}\right\}\left(\alpha_{n} \uparrow \rightarrow \infty\right)$ and Abel's method. Here we suppose that $\left\{\alpha_{n}\right\}$ is a monotone sequence converging to a real number $\alpha$ and consider inclusion relations of the form $C\left\{\alpha_{n}\right\} \subseteq C(\alpha)$. We show (for example) that if $\left\{\alpha_{n}\right\}$ increases to $\alpha$ sufficiently rapidly then $C\left\{\alpha_{n}\right\} \equiv C(\alpha)$, and that otherwise $C\left\{\alpha_{n}\right\} \subset C(\alpha)$. In this latter case we show that for a certain class of sequences $\left\{\alpha_{n}\right\}$ the "gap" between $C\left\{\alpha_{n}\right\}$ and $C(\alpha)$ may be filled by certain well-known Nörlund matrices.

In addition to the matrices $C(\alpha)$ and $\left\{C \alpha_{n}\right\}$ defined above we require the lower triangular matrices $C(\alpha, \gamma)$ and $C\left(\left\{\alpha_{n}\right\}, \gamma\right)$ whose $(n, k)$ th elements are given, for

(3) Certain identities involving the binomial coefficients $\varepsilon_{n}(\alpha)$ which we use freely can be found in [7, p. 77]. 
$0 \leqq k \leqq n$ by $\varepsilon_{n-k}(\alpha-1) \varepsilon_{k}(\gamma) / \varepsilon_{n}(\alpha+\gamma)$ and $\varepsilon_{n-k}\left(\alpha_{n}-1\right) \varepsilon_{k}(\gamma) / \varepsilon_{n}\left(\alpha_{n}+\gamma\right)$ respectively.

2. In this section we prove the following two theorems.

THEOREM 1. If $\left\{\alpha_{n}\right\}$ is a nondecreasing sequence converging to a real number $\alpha(>0)$ then

(i) $C\left\{\alpha_{n}\right\} \subseteq C(\alpha)$,

(ii) $C\left\{\alpha_{n}\right\} \equiv C(\alpha)$ if and only if $\left\{\left(\alpha-\alpha_{n}\right) \log n\right\}$ is bounded.

THEOREM 2. If $\left\{\alpha_{n}\right\}$ is a nonincreasing sequence converging to a real number $\alpha(\geqq 0)$ then

(i) $C(\alpha) \subseteq C\left\{\alpha_{n}\right\}$,

(ii) $C(\alpha) \equiv C\left\{\alpha_{n}\right\}$ if $\left(\alpha_{n}-\alpha\right) \log n$ is bounded,

(iii) $C(\alpha) \subset C\left\{\alpha_{n}\right\}$ if $\left(\alpha_{n}-\alpha\right) \log n \rightarrow \infty$ as $n \rightarrow \infty$.

We require two lemmas.

LEMMA 1. If $\alpha_{p} \geqq 0(p=0,1, \cdots)$ and $\left\{\alpha_{p}\right\}$ is bounded

$$
\varepsilon_{n}\left(\alpha_{p}\right)=\frac{n^{\alpha_{p}}}{\Gamma\left(\alpha_{p}+1\right)}\left(1+O\left(\frac{1}{n}\right)\right),
$$

uniformly in $n$ and $p$.

See $\left[7\right.$, p. 77]. The proof given there is for constant sequences $\left\{\alpha_{n}\right\}$ but is easily seen to cover the present case.

LEMMA $2\left(^{4}\right)$. If $0 \leqq m \leqq n$, if $0<\alpha_{r} \leqq 1(r=0,1, \cdots)$ and if either (i) $\left\{\alpha_{r}\right\}$ is a nondecreasing sequence, or (ii) $\left\{\alpha_{r}\right\}$ and $\left\{r^{-1}\left(1-\alpha_{r}\right)\right\}(r \geqq 1)$ are nonincreasing sequences, then for any sequence $\left\{s_{n}\right\}$ there is an integer $p$ such that $0 \leqq p \leqq m$ and

$$
\left(\varepsilon_{n}\left(\alpha_{n}-1\right)\right)^{-1}\left|\sum_{v=0}^{m} \varepsilon_{n-v}\left(\alpha_{n}-1\right) s_{v}\right| \leqq\left(\varepsilon_{p}\left(\alpha_{p}-1\right)\right)^{-1}\left|\sum_{v=0}^{p} \varepsilon_{p-v}\left(\alpha_{p}-1\right) s_{v}\right| .
$$

Proof. The result is trivially true if $m=n$ or $m=0$ and we suppose that $0<m<n$. It is easily verified that for fixed $m$ and $n, \varepsilon_{n-v}\left(\alpha_{n}-1\right)\left(\varepsilon_{m-v}\left(\alpha_{m}-1\right)\right)^{-1}$ is a nondecreasing function of $v$ in the range $0 \leqq v \leqq m$.

Consequently there is a nonincreasing sequence $m_{1} m_{2}, \cdots$ of positive integers such that

$$
\begin{aligned}
& \left|\sum_{v=0}^{m} \varepsilon_{n-v}\left(\alpha_{n}-1\right) s_{v}\right|=\left|\sum_{v=0}^{m} \varepsilon_{n-v}\left(\alpha_{n}-1\right)\left(\varepsilon_{m-v}\left(\alpha_{m}-1\right)^{-1} \varepsilon_{m-v}\left(\alpha_{m}-1\right) s\right)_{v}\right| \\
& \leqq \varepsilon_{n}\left(\alpha_{n}-1\right)\left(\varepsilon_{m}\left(\alpha_{m}-1\right)\right)^{-1}\left|\sum_{v=0}^{m_{1}} \varepsilon_{m-v}\left(\alpha_{m}-1\right) s_{v}\right| \\
& \leqq \varepsilon_{n}\left(\alpha_{n}-1\right)\left(\varepsilon_{m_{k}}\left(\alpha_{m_{k}}-1\right)\right)^{-1}\left|\sum_{v=0}^{m_{k+1}} \varepsilon_{m_{k}-v}\left(\alpha_{m_{k}}-1\right) s_{v}\right|
\end{aligned}
$$

(4) This lemma and its proof are given in the case of constant sequences $\left\{a_{r}\right\}$ by Bosanquet [3, Lemma 7]. 
Since the sequence $\left\{m_{k}\right\}$ is nonincreasing there is an integer $\rho$ such that $m_{\rho+1}=m_{\rho}=p$ (say) and in this case we have $0 \leqq p \leqq m$ and

$$
\left|\sum_{v=0}^{m} \varepsilon_{n-v}\left(\alpha_{n}-1\right) s_{v}\right| \leqq \varepsilon_{n}\left(\alpha_{n}-1\right)\left(\varepsilon_{p}\left(\alpha_{p}-1\right)\right)^{-1}\left|\sum_{v=0}^{p} \varepsilon_{p-v}\left(\alpha_{p}-1\right) s_{v}\right|
$$

which is the required result.

We also note that

$$
C\left\{\alpha_{n}\right\}=C\left(\left\{\alpha_{n}-\alpha\right\}, \alpha\right) C(\alpha) \text {. }
$$

Proof of Theorem 1. (i) We have to show that $s_{n} \rightarrow s C\left\{\alpha_{n}\right\}$ implies $s_{n} \rightarrow s C(\alpha)$, and since both $C\left\{\alpha_{n}\right\}$ and $C(\alpha)$ are regular matrices it is sufficient to obtain the result when $s=0$. It is also clear, from Theorem A(ii) that we may suppose $\alpha_{n} \neq \alpha(n=0,1, \cdots)$. Consequently from (2.1) it is sufficient to show that $t_{n} \rightarrow 0$ $C\left(\left\{\alpha_{n}-\alpha\right\}, \alpha\right)$ implies $t_{n}=o(1)$, or, writing $t_{-1}=0, x_{v}=\varepsilon_{v-1}(\alpha) t_{v-1}-\varepsilon_{v}(\alpha) t_{v}$ that

$$
\sum_{v=0}^{n} \varepsilon_{n-v}\left(\alpha_{n}-\alpha\right) x_{v}=o\left(\varepsilon_{n}\left(\alpha_{n}\right)\right)
$$

implies

$$
\left(t_{n} \varepsilon_{n}(\alpha)=\right) \sum_{v=0}^{n} x_{v}=o\left(\varepsilon_{n}(\alpha)\right) .
$$

Since $\left(\varepsilon_{n-v}\left(\alpha_{n}-\alpha\right)\right)^{-1}$ is an increasing function of $v$ in the range $0 \leqq v \leqq n$ we have, using Lemma 2 (case (i) with $\alpha_{n}$ replaced by $\alpha_{n}-\alpha-1$ )

$$
\begin{aligned}
\left|\sum_{v=0}^{n} x_{v}\right| & =\left|\sum_{v=0}^{n}\left(i_{n-v}\left(\alpha_{n}-\alpha\right)\right)^{-1} \varepsilon_{n-v}\left(\alpha_{n}-\alpha\right) x_{v}\right| \\
& \leqq 2 \max _{0 \leqq p \leqq n}\left|\sum_{v=0}^{p} \varepsilon_{n-v}\left(\alpha_{n}-\alpha\right) x_{v}\right| \\
& \leqq 2 \max _{0 \leqq p \leqq r} \max _{0 \leqq k \leqq p} \frac{\varepsilon_{n}\left(\alpha_{n}-\alpha\right)}{\varepsilon_{k}\left(\alpha_{k}-\alpha\right)}\left|\sum_{v=0}^{k} \varepsilon_{k-v}\left(\alpha_{k}-\alpha\right) x_{v}\right| \\
& =o\left(n^{\alpha_{n}}\right),
\end{aligned}
$$

by (2.2) and Lemma 1 , so that (2.3) holds.

To prove (ii) it is sufficient, in view of (2.1), to show that the matrix $C\left(\left\{\alpha_{n}-\alpha\right\}, \alpha\right)$ is regular if and only if $\left(\alpha-\alpha_{n}\right) \log n=O(1)$. Now $\sum_{v=0}^{n} \varepsilon_{n-v}\left(\alpha_{n}-\alpha-1\right) \varepsilon_{v}(\alpha)$ $=\varepsilon_{n}\left(\alpha_{n}\right)$ and it is easily verified, using Lemma 1 , that $\varepsilon_{n-v}\left(\alpha_{n}-\alpha-1\right) \varepsilon_{v}(\gamma)$ $\cdot\left(\varepsilon_{n}\left(\alpha_{n}\right)\right)^{-1}=o(1)$ for $v=0,1, \cdots$. Hence $C\left(\left\{\alpha_{n}-\alpha\right\}, \alpha,\right)$ is regular if and only if

$$
\begin{aligned}
\sum_{\nu=0}^{n}\left|\varepsilon_{n-v}\left(\alpha_{n}-\alpha-1\right)\right| \varepsilon_{v}(\alpha) & =2 \varepsilon_{n}(\alpha)-\sum_{\nu=0}^{n} \varepsilon_{n-v}\left(\alpha_{n}-\alpha-1\right) \varepsilon_{v}(\alpha) \\
& =2 \varepsilon_{n}(\alpha)-\varepsilon_{n}\left(\alpha_{n}\right) \\
& =O\left(\varepsilon_{n}\left(\alpha_{n}\right)\right)
\end{aligned}
$$


i.e., using Lemma 1, if and only if $\left(\alpha-\alpha_{n}\right) \log n=O(1)$.

Proof of Theorem 2. A straightforward calculation shows that $C\left(\left\{\alpha_{n}-\alpha\right\}, \alpha\right)$ is regular and (i) follows.

To prove (ii) we write $\beta_{n}=\alpha_{r}-\alpha$ and consider first the case when $\left\{\beta_{n}\right\}$ and $\left\{\left(1-\beta_{n}\right) / n\right\}$ are decreasing sequences. In this case it follows from Lemma 2 that if $0 \leqq m \leqq n$

$$
\begin{aligned}
\left(\varepsilon_{n}\left(\alpha_{n}\right)\right)^{-1} & \left|\sum_{v=0}^{m} \varepsilon_{n-v}\left(\beta_{n}-1\right) \varepsilon_{v}(\alpha) s_{v}\right| \\
& \leqq \max _{0 \leqq p \leqq m}\left(\varepsilon_{p}\left(\alpha_{p}\right)\right)^{-1}\left|\sum_{v=0}^{p} \varepsilon_{p-v}\left(\beta_{p}-1\right) \varepsilon_{v}(\alpha) s_{v}\right|
\end{aligned}
$$

It follows $[6$, p. 75$]$ that if $s_{n}=o(1) C\left(\left\{\beta_{n}\right\}, \alpha\right)$ then

$$
s_{k} \sup _{n \geqq k} \varepsilon_{n-k}\left(\beta_{n}-1\right) \varepsilon_{k}(\alpha)\left(\varepsilon_{n}\left(\alpha_{n}\right)\right)^{-1}=o(1) \text { as } k \rightarrow \infty \text {. }
$$

Now

$$
\varepsilon_{n-k}\left(\beta_{n}-1\right)=\frac{n(n-1) \cdots(n-k+1)}{\left(\beta_{n}+n-1\right)\left(\beta_{n}+n-2\right) \cdots\left(\beta_{n}+n-k\right)} \varepsilon_{n}\left(\beta_{n}-1\right)
$$

and it is easily verified that, since $\left\{\left(1-\beta_{n}\right) / n\right\}$ decreases, $\left\{(n-p) /\left(\beta_{n}+n-p-1\right)\right\}$ is a decreasing sequence for $n \geqq k$ and $p=0,1, \cdots, k-1$. Moreover, since $\left\{\beta_{n}\right\}$ decreases, $\left\{\varepsilon_{n}\left(\beta_{n}-1\right)\left(\varepsilon_{n}\left(\alpha_{n}\right)\right)^{-1}\right\}$ decreases. Consequently, for each fixed $k$, $\left\{\varepsilon_{n-k}\left(\beta_{n}-1\right)\left(\varepsilon_{n}\left(\alpha_{n}\right)\right)^{-1}\right\}$ decreases for $n \geqq k$ and so

$$
\sup _{n \geqq k} \varepsilon_{n-k}\left(\beta_{n}-1\right) \varepsilon_{k}(\alpha)\left(\varepsilon_{n}\left(\alpha_{n}\right)\right)^{-1}=\varepsilon_{k}(\alpha)\left(\varepsilon_{k}\left(\alpha_{k}\right)\right)^{-1}
$$

Thus, in the special case being considered, if $s_{n}=o(1) C\left(\left\{\beta_{n}\right\}, \alpha\right)$, then $s_{k} \varepsilon_{k}(\alpha)\left(\varepsilon_{k}\left(\alpha_{k}\right)\right)^{-1}=o(1)$, i.e., by Lemma $1, s_{k}=o\left(k^{\beta_{k}}\right)$. In particular, if $\alpha_{n}=\alpha+K / \log n$ for some positive $K$ and all sufficiently large $n$, then $s_{n}=o(1) C\left(\left\{\beta_{n}\right\}, \alpha\right)$ implies $s_{n}=o(1)$, i.e., that $C\left(\left\{\beta_{n}\right\}, \alpha\right)$ is equivalent to convergence. Now if $\left(\alpha_{n}-\alpha\right) \log n=O(1)$ we have $\alpha_{n} \leqq \alpha+K / \log n$ for some positive $K$ and all sufficiently large $n$. It follows from Theorem A (iii) that $C\left(\left\{\alpha_{n}-\alpha\right\}, \alpha\right)$ is equivalent to convergence and in view of (2.1) this proves (ii).

To obtain (iii) we note that $C\left(\left\{\alpha_{n}-\alpha\right\}, \alpha\right)$ is regular and that, since we may assume without loss of generality that $\alpha_{n}-\alpha<1, \varepsilon_{n-v}\left(\alpha_{n}-\alpha-1\right) \varepsilon_{v}(\alpha)$ increases with $v$ for $0 \leqq v \leqq n$ so that, writing $C\left(\left\{\alpha_{n}-\alpha\right\}, \alpha\right)=\left(a_{n v}\right)$ we have

$$
\begin{aligned}
\sum_{\nu=0}^{\infty}\left|a_{n v+1}-a_{n v}\right| & =2 a_{n n}-a_{n 0} \\
& =o(1)
\end{aligned}
$$

as $n \rightarrow \infty$ by Lemma 1 since $\left(\alpha_{n}-\alpha\right) \log n \rightarrow \infty$. It follows [1(a), p. 130] that $C\left(\left\{\alpha_{n}-\alpha\right\}, \alpha\right)$ evaluates some divergent sequence, and (iii) follows. 
3. Let $r_{n}(h, k)(h, k$, real $n=0,1, \cdots)$ be defined by

$$
(1-x)^{-h-1}\left(\frac{\log (1-x)}{-x}\right)^{k}=\sum_{n=0}^{\infty} r_{n}(h, k) x^{n} \quad(|x|<1) .
$$

It is known that [7, p. 193]

$$
\begin{array}{ll}
r_{n}(h, k) \sim \frac{n^{h}}{\Gamma(h+1)}(\log n)^{k} & (h \neq-1,-2, \cdots) \\
r_{n}(h, k) \sim(-1)^{k-1}(|k|-1) ! k n^{h}(\log n)^{k-1} & (h=-1,-2, \cdots) .
\end{array}
$$

Let $p_{n}(k)=r_{n}(-1, k)$, and let $L(k)$ denote the Nörlund matrix $[5, p .64]$ generated by the sequence $\left\{p_{n}(k)\right\}$. If $k \geqq 0$ we have $p_{n}(k) \geqq 0(n=1,2, \cdots)$ and $p_{0}(k)=1$. Moreover from (3.1) and (3.2)

and from (3.3)

$$
\sum_{v=0}^{n} p_{n}(k)=r(0, k) \sim(\log n)^{k}
$$

$$
p_{n}(k) \sim \frac{k(\log n)^{k-1}}{n}
$$

so that $[5$, p. 64$]$ if $k \geqq 0, L(k)$ is regular. The matrix $L(1)$ generates the harmonic means of M. Riesz and the matrices $L(k)$ may be regarded as iterates of $L(1)$ in the same sense that the matrices $C(\alpha)$ are iterates of $C(1)$.

We prove next

THEOREM 3. If $\alpha \geqq 0$ and $\alpha_{n}-\alpha=k \log \log n / \log n$ for sufficiently large values of $n$, where $k \geqq 0$, then $C\left\{\alpha_{n}\right\} \equiv L(k) C(\alpha)$.

We require some further lemmas.

LEMMA $3\left({ }^{5}\right)$. Suppose that $0<p_{n} \leqq K(n=0,1, \cdots)$, where $K$ is independent of $n$, and that the sequence $\left\{q_{n}\right\}$ is defined by

$$
q_{0} p_{0}=1, q_{n} p_{0}+q_{n-1} p_{1}+\cdots+q_{0} p_{n}=0 \quad(n=1,2, \cdots) .
$$

Suppose further that there is an integer $N(\geqq 0)$ such that

Then for any sequence $\left\{s_{v}\right\}$

$$
\begin{aligned}
q_{v} & \geqq 0, \quad v \geqq N, \\
\sum_{r=0}^{v} q_{v} \geqq 0, & v \geqq N .
\end{aligned}
$$

$$
\left|\sum_{v=0}^{m} p_{n-v} s_{v}\right| \leqq H \max _{0 \leqq k \leqq m}\left|\sum_{v=0}^{k} p_{k-v} s_{v}\right| \quad(0 \leqq m \leqq n)
$$

where $H$ is independent of $n$.

(5) This is an extension of some known results cf. [6, p. 43 and p. 128]. 
Proof. Since the result is clearly true if $m=n$ we suppose that $0 \leqq m<n$. From the definition of the sequence $\left\{q_{n}\right\}$ we have

for $v=0,1, \cdots$.

$$
\begin{aligned}
s_{v} & =\sum_{r=0}^{v} q_{v-r} \sum_{t=0}^{r} p_{r-t} s_{r} \\
& =\sum_{r=0}^{v} q_{v-r} t_{r} \quad \text { (say), }
\end{aligned}
$$

Consequently

$$
\begin{aligned}
\left|\sum_{v=0}^{m} p_{n-v} s_{v}\right| & =\left|\sum_{v=0}^{m} p_{n-v} \sum_{r=0}^{v} q_{v-r} t_{r}\right| \\
& =\left|\sum_{r=0}^{m} t_{r} \sum_{v=0}^{m-r} p_{n-r-v} q_{v}\right| \\
& \leqq \max _{0 \leqq r \leqq m}\left|t_{r}\right| \sum_{r=0}^{m}\left|\sum_{v=0}^{m-r} p_{n-r-v} q_{v}\right| .
\end{aligned}
$$

If $m \leqq N$

$$
\sum_{r=0}^{m}\left|\sum_{v=0}^{m-r} p_{n-r-v} q_{v}\right| \leqq K \sum_{r=0}^{N} \sum_{v=0}^{N}\left|q_{v}\right|=K \quad \text { (say). }
$$

If $m>N$ we write

$$
\sum_{r=0}^{m}\left|\sum_{v=0}^{m-r} p_{n-r-v} q_{v}\right|=\sum_{r=0}^{n-N}\left|\sum_{v=0}^{m-r} p_{n-r-v} q_{v}\right|+\sum_{r=m-N+1}^{m} \sum_{v=0}^{m-r} p_{n-r-v} q_{v} \mid \text {. }
$$

Now if $0 \leqq r \leqq m-N$ and $v>m-r$ we have $v>N$ so that $q_{v} \leqq 0$ by (3.4) and so for $0 \leqq r \leqq m-N$

$$
\sum_{v=0}^{m-r} p_{n-r-v} q_{v} \geqq \sum_{v=0}^{n-r} p_{n-r-v} q_{v}=0 .
$$

Consequently if $m>N$

$$
\begin{aligned}
\sum_{r=0}^{m}\left|\sum_{v=0}^{m-r} p_{n-r-v} q_{v}\right| & \leqq \sum_{r=0}^{m} \sum_{v=0}^{m-r} p_{n-r-v} q_{v}+2 \sum_{r=m-N+1}^{m}\left|\sum_{v=0}^{m-r} p_{n-r-v} q_{v}\right| \\
& \leqq \sum_{r=0}^{m} \sum_{v=0}^{m-r} p_{n-r-v} q_{v}+2 K N \sum_{v=0}^{N}\left|q_{v}\right| \\
& =\sum_{v=0}^{m} p_{n-v} \sum_{r=0}^{v} q_{v-r}+2 K^{\prime} \\
& \left.\leqq \sum_{v=0}^{n} p_{n-v} \sum_{r=0}^{v} q_{v}+2 K^{\prime} \quad \text { (by (3.5) since } m>N\right) \\
& =1+2 K^{\prime}=K^{\prime \prime} \quad \text { (say). }
\end{aligned}
$$


If we now choose $H=\max \left(K^{\prime}, K^{\prime \prime}\right)$ we have

$$
\sum_{r=0}^{m}\left|\sum_{v=0}^{m-r} p_{n-r-v} q_{v}\right| \leqq H
$$

and (3.6) follows from this and (3.7).

LEMMA 4. If $\gamma \geqq 0$ and if $\left\{\alpha_{n}\right\}$ satisfies either of the two conditions of Lemma 2 then $C\left\{\alpha_{n}\right\} \equiv C\left(\left\{\alpha_{n}\right\}, \gamma\right)$.

Proof. The case $\gamma=0$ being trivial we suppose that $\gamma>0$. It follows from Theorem A (iii) and known results $\left[2\right.$, Theorem 8] that $C\left(\left\{\alpha_{n}\right\}, \gamma\right) \subseteq C(1)$. Consequently if $s_{n}=o(1) C\left(\left\{\alpha_{n}\right\}, \gamma\right)$ and we write $\tau_{n}=\max _{0 \leqq v \leqq n}\left|\Sigma_{p=0}^{\gamma} s_{p}\right|$ we shall have $\tau_{n}=o(n)$. Putting $\rho_{n}=\left[n-\tau_{n}\right]$ we have

$$
\sum_{v=0}^{n} \varepsilon_{n-v}\left(\alpha_{n}-1\right) s_{v}=\sum_{v=0}^{p_{n}}+\sum_{\rho_{n}+1}^{n}=\sum_{1}+\sum_{2} \text { (say) }
$$

where, since $\varepsilon_{n-v}\left(\alpha_{n}-1\right)$ is an increasing function of $v$ for $0 \leqq v \leqq n$

$$
\begin{aligned}
\left|\sum_{1}\right| & \leqq 2 \varepsilon_{n-\rho_{n}}\left(\alpha_{n}-1\right) \tau_{n} \\
& =o\left(n^{\alpha_{n}}\right)
\end{aligned}
$$

by Lemma 1 . By Lemma 2 we have since $\left(\varepsilon_{v}(\gamma)\right)^{-1}$ decreases as $v$ increases

$$
\begin{aligned}
\left|\sum_{2}\right| & =\left|\sum_{v=\rho_{n}+1}^{n} \varepsilon_{n-v}\left(\alpha_{n}-1\right) \varepsilon_{v}(\gamma)\left(\varepsilon_{v}(\gamma)\right)^{-1} s_{v}\right| \\
& \leqq\left(\varepsilon_{\rho_{n}+1}(\gamma)\right)^{-1} \max _{\rho_{n+1} \leqq p \leqq n}\left|\sum_{v=0}^{p} \varepsilon_{n-v}\left(\alpha_{n}-1\right) \varepsilon_{v}(\gamma) s_{v}\right| \\
& \leqq\left(\varepsilon_{\rho_{n}+1}(\gamma)\right) \max _{\rho_{n}+1 \leqq p \leqq n} \max _{0 \leqq k \leqq p} \frac{\varepsilon_{n}\left(\alpha_{n}-1\right)}{\varepsilon_{k}\left(\alpha_{k}-1\right)}\left|\sum_{v=0}^{k} \varepsilon_{k-v}\left(\alpha_{k-1}\right) \varepsilon_{v}(\gamma) s_{v}\right| \\
& =o\left(n^{a_{n}}\right)
\end{aligned}
$$

by Lemma 1 . Consequently $s_{n}=o(1) C\left\{\alpha_{n}\right\}$.

Conversely if $s_{n}=o(1) C\left\{\alpha_{n}\right\}$ then, by Lemma 2 ,

$$
\begin{aligned}
\left|\sum_{v=0}^{n} \varepsilon_{n-v}\left(\alpha_{n}-1\right) \varepsilon_{v}(\gamma) s_{v}\right| \leqq 2 \varepsilon_{n}(\gamma) \max _{0 \leqq p \leqq n}\left|\sum_{v=0}^{p} \varepsilon_{n-v}\left(\alpha_{n}-1\right) s_{v}\right| \\
\leqq 2 \varepsilon_{n}(\gamma) \max _{0 \leqq p \leqq n} \max _{0 \leqq k \leqq p} \frac{\varepsilon_{n}\left(\alpha_{n}-1\right)}{\varepsilon_{k}\left(\alpha_{k}-1\right)} \\
\\
\cdot\left|\sum_{v=0}^{k} \varepsilon_{k-v}\left(\alpha_{k-1}\right) s_{v}\right| \\
=o\left(n^{\alpha_{n}+\gamma}\right),
\end{aligned}
$$

and the result follows. 
Proof of Theorem 4. It is clearly sufficient after (2.1) and Lemma 4 to show that $L(k) \equiv C\left\{\beta_{n}\right\}$ where $\beta_{n}=\alpha_{n}-\alpha=k \log \log n / \log n$ for $n \geqq 4$ and the initial values of $\beta_{n}$ are chosen so that $\left\{\left(1-\beta_{n}\right) / n\right\}$ is decreasing. (Alteration of a finite number of the values of $\beta_{n}$ clearly has no effect on the summability properties of $C\left\{\beta_{n}\right\}$.)

We first show that

$$
C\left\{\beta_{n}\right\} \subseteq L(k)
$$

Since both methods in (3.8) are regular it is sufficient to show that

$$
\sum_{v=0}^{n} \varepsilon_{n-v}\left(\beta_{n}-1\right) s_{v}=o\left((\log n)^{k}\right)
$$

implies

$$
\sum_{v=0}^{n} p_{n-v}(k) s_{v}=o\left((\log n)^{k}\right)
$$

We abbreviate $p_{n}(k)$ to $p_{n}$ for the rest of this proof. We then have, by Lemma 2 ,

$$
\begin{aligned}
\left|\sum_{v=0}^{n} p_{n-v} s_{v}\right| & =\left|\sum_{v=0}^{n} \rho_{n-v} \varepsilon_{n-v}\left(\beta_{n}-1\right) s_{v}\right| \\
& =o\left(n^{\beta_{n}}\right)\left\{\sum_{v=0}^{n-1}\left|\rho_{n-v}-\rho_{n-v-1}\right|+\rho_{0}\right\}
\end{aligned}
$$

where $\rho_{s}=p_{s}\left(\varepsilon_{s}\left(\beta_{n}-1\right)\right)^{-1}$. Now

$$
\frac{\rho_{s}}{\rho_{s+1}}=\frac{p_{s}}{p_{s+1}} \frac{\beta_{n}+s}{s+1}
$$

so that, by (3.3) and a routine calculation

$$
\rho_{s}-\rho_{s+1}=\beta_{n} \rho_{s+1} o\left(\frac{1}{s}\right)+\rho_{s+1} o\left(\frac{1}{s \log s}\right) .
$$

Using Lemma 1 and (3.3) we have

$$
\begin{aligned}
\beta_{n} \sum_{s=1}^{n} \rho_{s+1} O\left(\frac{1}{s}\right) & =\sum_{s=1}^{n} O\left(\frac{(\log s)^{k-1}}{s^{\beta_{n+1}}}\right) \\
& =O(1)
\end{aligned}
$$

while, if we write

$$
t_{s}=\sum_{r=s}^{\infty} \frac{(\log r)^{k-2}}{r^{2}}\left(=0\left(\frac{(\log s)^{k-2}}{s}\right)\right)
$$


and observe that $\left(\varepsilon_{s}\left(\beta_{n}-1\right)\right)^{-1}-\left(\varepsilon_{s+1}\left(\beta_{n}-1\right)\right)^{-1}=\left(\varepsilon_{s}\left(\beta_{n}\right)\right)^{-1}$ we have

$$
\begin{aligned}
\sum_{s=2}^{n} \rho_{s+1} O\left(\frac{1}{s \log s}\right) & =O\left(\sum_{s=2}^{n}\left(\varepsilon_{s}\left(\beta_{n}-1\right)\right)^{-1} \frac{(\log s)^{k-2}}{s^{2}}\right) \\
& =O\left(\sum_{s=2}^{n} t_{s}\left(\varepsilon_{s}\left(\beta_{n}\right)\right)^{-1}+t_{n}\left(\varepsilon_{n}\left(\beta_{n}-1\right)\right)^{-1}\right) \\
& =O\left(\sum_{s=1}^{n} \frac{(\log s)^{k-2}}{s^{\beta_{n+1}}}\right)+O\left(\frac{(\log n)^{k-2}}{\beta_{n} n^{\beta_{n}}}\right) \\
& =O(1) .
\end{aligned}
$$

It follows that

$$
\sum_{s=1}^{n}\left|\rho_{s}-\rho_{s+1}\right|=O(1)
$$

Using this and (3.11) we obtain (3.10). To obtain the inclusion reverse to (3.8) we suppose that $s_{n}=o(1) L(k)$. If $q_{n}=q_{n}(k)=r_{n}(-1,-k)$ then $p_{0} q_{0}=1$, $p_{0} q_{n}+p_{1} q_{n-1}+\cdots+p_{n} q_{0}=0(n \geqq 11)$. Moreover by (3.2)

$$
q_{n} \sim-k \frac{(\log n)^{k-1}}{n} \text { and } \sum_{v=0}^{n} q_{v}=r_{n}(0,-k) \sim(\log n)^{-k} \text {. }
$$

Consequently $\left\{p_{n}\right\}$ and $\left\{q_{n}\right\}$ satisfy the conditions of Lemma 3 and so we have

$$
\begin{aligned}
\left|\sum_{v=0}^{n} \varepsilon_{n-v}\left(\beta_{n-1}\right) s_{v}\right| & =\left|\sum_{v=0}^{n} \tau_{n-v} p_{n-v} s_{v}\right| \\
& \leqq \max _{0 \leqq k \leqq n}\left|\sum_{v=0}^{k} p_{k-v} s_{v}\right|\left\{\sum_{v=0}^{n-1}\left|\tau_{n-v}-\tau_{n-v-1}\right|+\tau_{0}\right\} \\
& =o(\log n)^{k}\left\{\sum_{v=1}^{n}\left|\tau_{s}-\tau_{s+1}\right|+\tau_{0}\right\}
\end{aligned}
$$

where $\tau_{s}=\varepsilon_{s}\left(\beta_{n}-1\right) / p$. The required result now follows by arguments similar to those used above.

A counterpart to Theorem 3 is:

THEOREM 4. If $\alpha>0$ and $\alpha-\alpha_{n}=k \log \log n / \log n$ for sufficiently large values of $n$, where $k \geqq 0$, then $L(k) C\left\{\alpha_{n}\right\} \equiv C(\alpha)$.

The proof is similar to that of Theorem 3 and we omit the details.

We remark in conclusion that the form of Theorem 3 and 4 suggests that if we write $\beta_{n}=\left|\alpha-\alpha_{n}\right|$ and $H$ for the Nörlund method generated by the sequence $\left\{(n+1)^{\beta_{n+1}}-n^{\beta_{n}}\right\}$ then $C\left\{\alpha_{n}\right\} \equiv H C(\alpha)$ or $H C\left\{\alpha_{n}\right\}=C(\alpha)$ according as $\left\{\alpha_{n}\right\}$ is decreasing or increasing. 


\section{REFERE NCES}

1. R. P. Agnew, Inclusion relations among methods of summability compounded from given matrix methods, Ark. Mat. 2 (1952), 361-374.

1(a). - A simple sufficient condition that a method of summability be stronger than convergence, Bull. Amer. Math. Soc. 52 (1946), 128-132.

2. D. Borwein, On some methods of summability, Quart. J. Math. Oxford Ser. (2) 9 (1958), 310-316.

3. L. S. Bosanquet, Note on convergence and summability factors III, Proc. London Math. Soc. (2) 50 (1949), 482-496.

4. G. G. Lorentz, Direct theorems on methods of summability, Canad. J. Math. 1 (1949), 305-319.

5. G. H. Hardy, Divergent series, Oxford, at the Clarendon Press, 1949.

6. K. Zeller, Theorie der Limitierungsverfahren, Springer, Berlin, 1958.

7. A. Zygmund, Trigonometric series. I, 2nd. ed. Cambridge Univ. Press, New York, 1960.

UNIVERSITY OF ABERDEEN,

ABERdEEN, SCOTLAND 